\title{
Prediction of Soybean Price Trend via a Synthesis Method With Multistage Model
}

\author{
Zhiling Xu, Northeast Agricultural University, Harbin, China \\ Hualing Deng, Northeast Agricultural University, Harbin, China \\ Qiufeng Wu, Northeast Agricultural University, Harbin, China \\ (iD) https://orcid.org/0000-0002-4787-2549
}

\begin{abstract}
Soybean is an important crop, so it is very important to forecast soybean price trend, which can stabilize the market. This paper presents a synthesis method with multistage model (SMwMM) in order to identify and forecast soybean price trend in China. In the previous work, Toeplitz inverse covariance-based clustering (TICC) has been applied to cluster the prices of four variables. The research has found that there are four patterns in soybean market price, which could be explained by economic theory. This paper considers four patterns as market risk levels. Based on the clustering results, the authors used long short-term memory (LSTM) to forecast the prices of these four variables. Multivariate long short-term memory (MLSTM) is then used to classify soybean price to determine level of risk. Experimental results show that (1) the LSTM model has achieved great fitting effect and high prediction accuracy and (2) the performance of MLSTM-FCN and MALSTM-FCN is better than that of LSTM-FCN and ALSTM-FCN. Furthermore, MALSTM-FCN had a higher accuracy than MLSTM-FCN, which reached 76.39\%.
\end{abstract}

\section{KEYWORDS}

Forecast, Long Short-Term Memory, Multivariate Long Short-Term Memory, Soybean Price

\section{INTRODUCTION}

The soybean is an important source of high quality protein for human beings and an important raw material of edible oil. The soybean products are hard to be replaced as part of the human diet (He et al., 2017). In addition, the breeding industry needs a large amount of feed, which is mainly composed of corn and soybean. The corn provides sugar and the soybean provides protein. Therefore, soybean price directly affects soybean production, farmer income, animal husbandry cost and the stability of agricultural products market (Li, 2014).

From the economic angle, soybean plays such an important role in breeding industry that soybean price affects meat price. In addition, the instability of soybean price brings significant risks to other grains price (Xu \& Ma, 2018). Therefore, the fluctuation of soybean price affects the development of agriculture, the quality of consumer life and the overall stability of market economy (Qian, 2017). Timely and accurate prediction for soybean price trend enables the government to make corresponding decisions in time and stabilize the market (Jiang, 2018). However, the drastic change of international 
soybean market leads to the continuous fluctuation of domestic soybean price in recent years. In this context, it is more important to predict soybean market price trend in China.

This paper aims at predicting soybean price trend in China. Predicting soybean price trend in China needs firstly determines different soybean price trend, that is, "Pattern of Soybean Price (PSP)" (Deng\&Sun, 2019). Then forecast soybean price trend based on PSP classes and predicted soybean prices. Based on these analyses, the prediction of soybean price trend consists of three steps. Firstly, this paper cites the research results from Deng and Sun (2019), that is, there are four patterns in soybean price. Secondly, four variables related to soybean price are predicted respectively. The four variables are the soybean purchase price, corn market price, soybean futures price, and the soybean oil futures price. The second step is formalized as univariate time series prediction problem. Finally, the future soybean price trend is predicted according to the four kinds of predicted price. The third step is formalized as a multivariate time series classification problem, that is, the predicted prices are classified to determine which soybean price model they are in.

This paper proposes a Synthesis Method with Multistage Model (SMwMM), including clustering, prediction and classification, in order to predict soybean price trend. Firstly, this paper cites the research results from Deng and Sun (2019), that is, there are four patterns in soybean price. Deng and Sun (2019) used Toeplitz Inverse Covariance-based Clustering (TICC) (Hallac et al., 2017) to cluster the multivariate time series data set composed of four variables, including soybean purchase price, corn market price, soybean futures price and soybean-oil futures price. These four variables have a greater impact on soybean prices (Deng \& Sun, 2019). Therefore, this paper also uses these four variables to conduct experiments in order to predict the soybean price trend more accurately. Then Long Short-Term Memory (LSTM) (Graves et al., 2012) is applied to forecast soybean purchase price, corn market price, soybean futures price and soybean oil futures price, respectively. Finally, this paper forecasts soybean price trend via Multivariate Attention Long Short-Term Memory Fully Convolutional Networks (MALSTM-FCN) (Karim et al., 2019), that is, classify price to determine which pattern the soybean price is in. In general, a new combination is proposed to predict soybean price trend by using the existing models. This combination is named SMwMM. Therefore, the contribution of this paper lies in the establishment of new soybean risk early-warning model. Compared with the traditional early-warning methods, this paper takes the discovered pattern as the early-warning alarm degree, which can avoid the subjectivity of artificial selection. This is helpful for more accurate prediction and early warning of soybean price. At the same time, it is also a new idea for agricultural monitoring and early warning, which provides methods and basis for the establishment of risk warning models for other agricultural products in China.

The remainder of this paper is organized as follows: Section 2 describes the literature review of soybean price prediction and classification algorithm. Section 3 briefly introduces structure of LSTM and the details of MALSTM, and explains the procedure of predicting soybean price trend via SMwMM. Experimental results are discussed in Section 4. Section 5 explains conclusions and major contributions.

\section{RELATED WORKS}

At present, there are a lot of researches about soybean price prediction at home and abroad. For example, Wang et al. (2016) derived the theoretical model of the optimal confidence interval to simulate the optimal interval forecast of soybean meal and non-GMO soybean futures price. David et al. (2017) applied the Auto Regressive Fractionally Integrated Moving Average (ARFIMA) model to predict soybean price. He et al. (2017) proposed an support vector regression based on adaptive particle swarm optimization (APSO_SVR) model to predict soybean price in China. Zhang et al. (2018) applied a quantile regression-radial basis function (QR-RBF) neural network model to predict of soybean price in China. Drachal (2019) proposed a new Bayesian model combination schemes for analysis of soybean price. These researches mentioned above are about to forecast univariate time 
series. In this paper, a new model combination is proposed, which uses multivariate time series to predict soybean price trend, considering four variables that have great influence on soybean price. Therefore, the warning model proposed in this paper can more accurately grasp the soybean price trend. It provides a new idea for soybean price prediction, and also provides a method and basis for the establishment of agricultural product risk warning model.

In this paper, the soybean price trend prediction is divided into three steps. The first step cited the previous research results, namely, TICC was used to cluster soybean prices into four patterns (Deng\&Sun, 2019). In the second step, LSTM is applied to predict the four time series. LSTM algorithm is well known by researchers in the area of machine learning. Meanwhile, many studies have proved the superiority of LSTM in price prediction, such as Peng et al. (2019) and Baek\&Kim (2018). The third step needs to classify multivariate time series more effectively.

In recent years, several time series classification algorithms have been developed in succession. Distance based methods along with K-Nearest Neighbors (KNN) have been proven to be successful in classifying multivariate time series (Orsenigo \& Vercellis, 2010). Plenty of research indicates Dynamic Time Warping (DTW) as the best distance based measure to use along with KNN (Seto et al., 2015). In addition to distance based metrics, Hidden State Conditional Random Field (HCRF) and Hidden Unit Logistic Model (HULM) are two successful feature based algorithms that have led to state of the art results on various benchmark datasets (Pei et al., 2018). Traditional models, such as Naive Logistic model (NL) and Fisher Kernel Learning (FKL) (Jaakkola et al., 2000), showed strong performance on a wide variety of time series classification problems. Another common approach for multivariate time series classification is to apply dimensional reduction techniques or concatenate all dimensions of a multivariate time series into a univariate time series, such as Symbolic Representation for Multivariate Time Series (SMTS) (Baydogan et al., 2014), Learned Pattern Similarity (LPS) (Baydogan et al., 2015), Ultra-Fast Shape (UFS) (Wistuba et al., 2015), Auto-Regressive (AR) kernel (Cuturi \& Doucet, 2011) and Auto-Regressive Forests (Tuncel \& Baydogan, 2018). Deep learning has also yielded promising results for multivariate time series classification. In 2014, Zheng et al. (2014) proposed Multi-Channel Deep Convolutional Neural Network (MC-DCNN) for multivariate time series classification.

The model used in this paper requires minimal preprocessing. Karim et al. (2019) tested MALSTM-FCN on 35 datasets, obtaining strong performance. MALSTM-FCN and Multivariate LSTM Fully Convolutional Networks (MLSTM-FCN) are beneficial in various multivariate time series classification tasks, such as activity recognition, or action recognition (Karim et al., 2019). MLSTM-FCN and MALSTM-FCN enhance the classification ability of LSTM Fully Convolutional Network (LSTM-FCN) and Attention LSTM-FCN (ALSTM-FCN), respectively. Therefore, this paper uses MALSTM-FCN for multivariate time series classification, compared with MLSTM-FCN, LSTM-FCN (Karim et al., 2018) and ALSTM-FCN (Karim et al., 2018). LSTM-FCN has been used in industrial installation processes (Patxi et al., 2020) and video detection (Wu et al., 2020). To our knowledge, this is the first application of MALSTM-FCN and MLSTM-FCN in agriculture.

\section{METHODS AND MATERIALS}

\section{Procedure of Prediction of Soybean Price Trend}

The main target of this paper is to forecast soybean price trend. First, pattern of soybean price need to be determined. Then forecast the four variables respectively and finally classify data to determine which pattern the price is in. This is the procedure of predicting soybean price trend. As shown in Figure 1.

Deng and Sun (2019) used TICC to cluster the multivariate time series dataset composed of four variables. The four variables are closely related to soybean prices. Deng and Sun (2019) concluded that there are four patterns in soybean price. This paper cites this conclusion. This paper takes these four patterns as the warning risk levels in this paper. In order to make the results more accurate, 


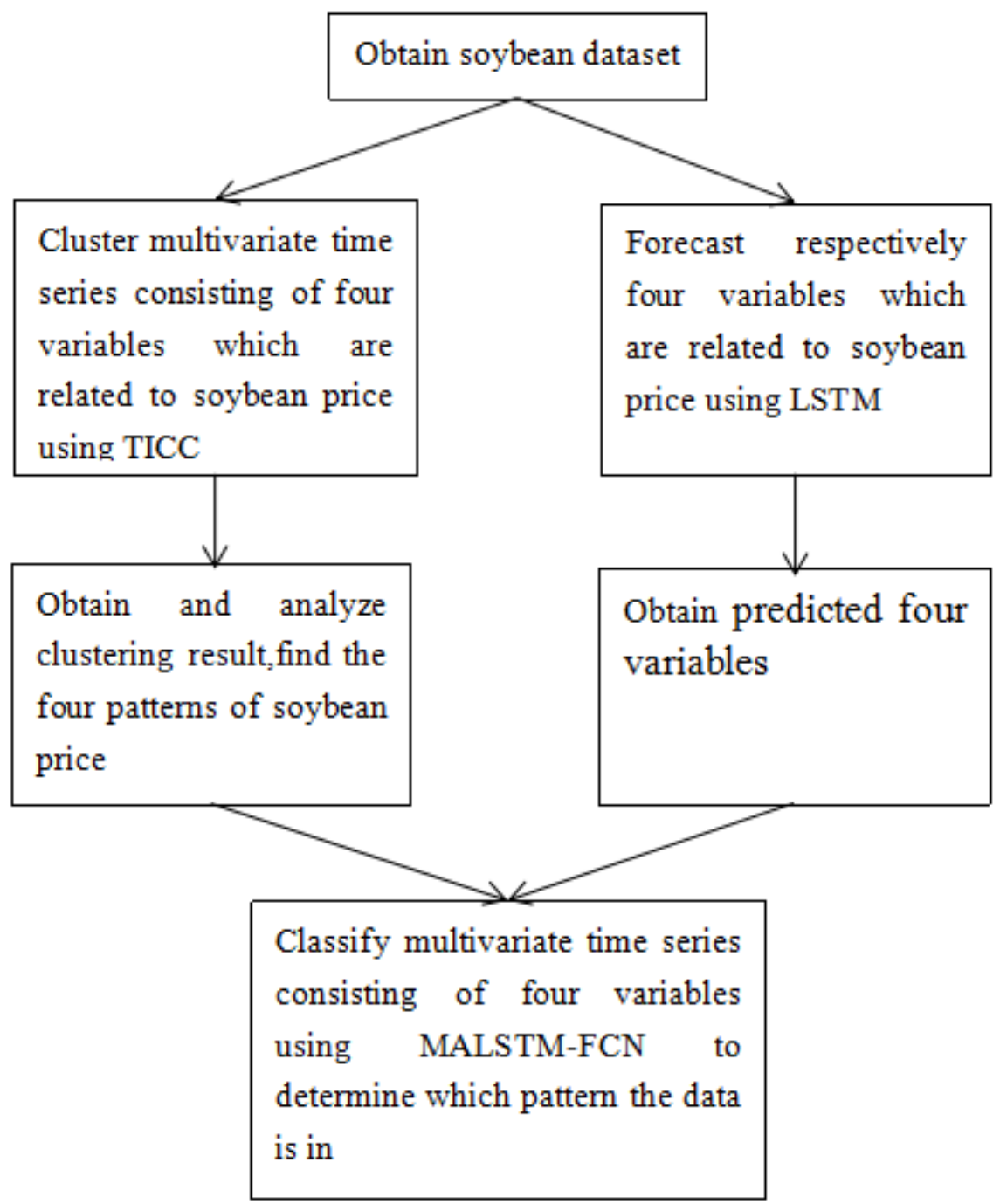

this paper selects the same four factors as experimental data, namely soybean purchase price, corn market price, soybean futures price and soybean oil futures price. Then, LSTM is applied to forecast soybean purchase price, corn market price, soybean futures price and soybean oil futures price and experiment showed higher accuracy. Finally, MALSTM-FCN is applied to classify the multivariate time series dataset and achieves high accuracy. That is to effectively judge the soybean market price will be in which market risk level.

\section{Soybean Price Pattern Discovery Via TICC}

Deng and Sun (2019) used TICC to cluster the multivariate time series composed of four variables. These four variables are soybean purchase price, corn market price, soybean futures price and soybean oil futures price, which are closely related to soybean price. The clustering results show that there are four patterns in soybean price. These four patterns correspond to the four phases of the economic cycle. The four stages are boom, recession, depression and recovery. This paper quotes conclusion 
Figure 2. The repeated module in the LSTM contains four interacting layers

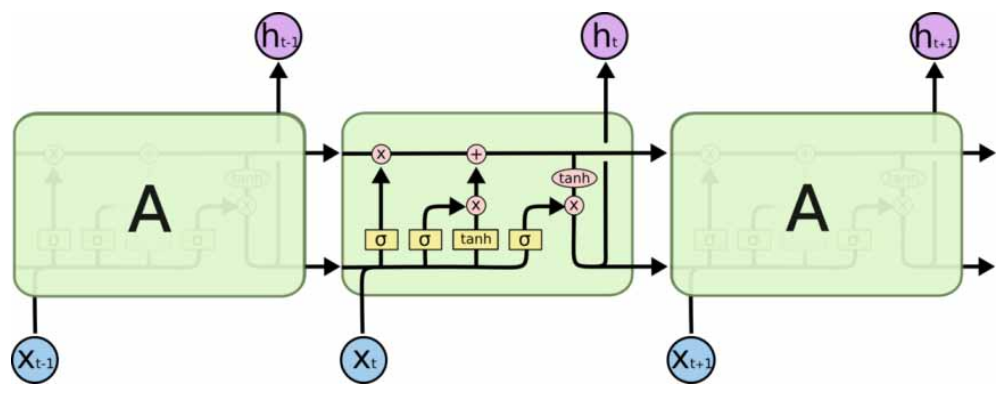

of Deng and Sun (2019), that is, there are four models in soybean price. This is the first step in this paper, which prepares for the classification in third step.

\section{Prediction of Soybean Price Sequences Via LSTM}

Long short-term memory is a special kind of Recurrent Neural Network, which can learn long time dependencies. The main feature is to solve the problem of gradient disappearance and gradient explosion during long sequence training by integrating the gating function into its state dynamics (Hochreiter \& Schmidhuber, 1997).

Compared with RNN, the repeated modules of LSTM has a different structure. There are four layers instead of a single layer, interacting in a very special way. Modules have smarter components than classical neurons and a memory for recent sequences. Modules contain the state of the management blocks and gates for output. Blocks operate on input sequences, and each gate in a block uses an s-shaped activation unit to control whether or not they are triggered. Each unit is like a small state machine, and each door in the unit has weights learned during training (Figure 2).

\section{Classification of Soybean Price Sequences Via MALSTM-FCN}

\section{Temporal Convolutions}

The MALSTM-FCN model (Karim et at., 2019) uses the Temporal Convolutional Network (TCN) as a feature extraction module of FCN branch. In general, a basic convolution block contains a convolution layer, which is accompanied by a batch normalization (Ioffe \& Szegedy, 2015). The batch normalization is followed by an activation function of either a Rectifified Linear Unit or a Parametric Rectifified Linear Unit (Trottier et al., 2016).

\section{Attention Mechanism}

Bahanau et al. (2014) proposed attention mechanism. Attention mechanism imposes conditions on a context vector on the target sequence. The context vector depends on a sequence of annotation, where the encoder maps the input sequence. Each annotation contains information on the whole input sequence, with an emphasis on the area around the $i$-th information of the input sequence (Karim et al., 2019).

\section{Squeeze and Excite Block}

$\mathrm{Hu}$ et al. (2017) proposed an extruded exciter block as a transformation unit. $\mathrm{Hu}$ et al. (2017) modeled the channel interdependencies to adjust the filter responses in two steps which are squeeze and excitation. The squeeze operation exploits the contextual information outside the local receptive field by using a global average pool to generate channel-wise statistics. The transformation output is shrunk through spatial dimensions to compute the channel-wise statistics. For temporal sequence data, the transformation output, is shrunk through the temporal dimension to compute the channel- 
wise statistics. The aggregated information from the squeeze operation is followed by an excite operation, whose objective is to capture the channel-wise dependencies. To achieve this, a simple gating mechanism is applied with a sigmoid activation (Karim et al., 2019).

\section{Network Architecture and Network Input of MALSTM-FCN}

MALSTM-FCN used in this paper is a new algorithm for multivariate time series classification. Karim et al. (2019) proposed MLSTM-FCN and MALSTM-FCN, which enhance respectively the classification ability of LSTM-FCN and ALSTM-FCN.

MLSTM-FCN and MALSTM-FCN comprise of a fully convolutional block and a LSTM block. The fully convolutional block contains three temporal convolutional blocks for feature extraction. Each convolutional block contains a convolutional layer, with filter size of 128 or 256, with a momentum of 0.99 and epsilon of 0.001 , and then batch normalization. The batch normalization layer is activated by the Rectified Linear Units (ReLU). In addition, the first two convolutional blocks have squeeze and excite blocks. The final temporal convolutional block is followed by a global average pooling layer.

Multivariate time series input, on the other hand, is passed through a dimension shuffle layer followed by the LSTM block. The LSTM block in method used in this paper is the same as block from the LSTM-FCN or ALSTM-FCN models, including an LSTM layer or an Attention LSTM layer, followed by an output layer. If there is missing data in the data set, we usually fill the data with the zero vector at the end to make them consistent in size, which requires to use a mask to skip time steps for which we have no information prior to the LSTM or Attention LSTM layer to.

Depending on the data set, the input to the fully convolutional block and LSTM block are different. The input to a fully convolution block is a multivariate time series with $N$ time steps, each of which has $M$ variables. If there is a time series with $M$ variables and $N$ time steps, the fully convolution block will receive such data. The input to the LSTM can vary depending on the application of dimensional shuffle. The dimension shuffle transforms the temporal dimension of the input data. If the input of LSTM does not go through dimension shuffle, the LSTM will need $N$ time steps to process $M$ variables at each time step. However, if dimension shuffle is applied, the LSTM will need $M$ time steps to process $N$ variables. In other words, when the number of variables $M$ is less than the time steps $N$, dimension shuffle improves the efficiency of the model. In the model used in this paper, dimension shuffle is only used when the number of time steps $N$ is greater than the number of variables $M$.

\section{EXPERIMENTAL RESULTS AND DISCUSSION}

According to the research from Deng and Sun (2019), soybean market price is affected by soybean purchase price, corn market price, soybean futures price and soybean-oil futures price. Therefore, this paper also uses these four variables to conduct experiments in order to predict the soybean price trend more accurately. Heilongjiang soybean price is selected as the experimental object, because Heilongjiang is the major soybean production area. Experimental data are obtained from Dalian Commodity Exchange (http://www.dce.com.cn/DCE/DCE_PAGE_KEY/index.html), Heilongjiang agricultural information net (http://www.hljagri.gov.cn/ddw/scbj/) and pig price net (http://www. zhujiage.com.cn/special/hlj_dadoujiage.html). The range of data covers all working days from July 15, 2016 solstice to December 31, 2017 (358 items in total). This paper obtains data through writing a web crawler program. And then clean and calculate the text crawled down. It's worth noting that there are missing data in the data. To solve this problem, this paper uses Lagrange interpolation to fill in the missing data.

This paper cites the research results from Deng and Sun (2019), that is, there are four patterns in soybean price. Deng and Sun (2019) used Toeplitz Inverse Covariance-based Clustering (TICC) to cluster the multivariate time series data set composed of four variables (Deng \& Sun, 2019). Deng and Sun (2019) made a reasonable assumption for each pattern, that is, pattern 1 is recession, pattern 
2 is boom, pattern 3 is recovery, and pattern 4 is depression. On this basis, this paper takes these four patterns as the warning risk level.

\section{Experiments on Prediction of Soybean Price}

This paper uses the LSTM model with "memory function" to forecast the data of the four variables, separately. There are some commonly used models for predicting time series, such as Artificial Neural Network (ANN) (Jain et al., 1996), Recurrent Neural Network (RNN) (Ah C.T., 1998) and Gated Recurrent Unit (GRU) (Cho et al., 2014). To highlight the superiority of LSTM, this paper applies ANN, RNN and GRU as comparison models. To evaluate the performance of models, all models uses the same parameter settings. $70 \%$ of the data sets are used to train model, and the remaining $30 \%$ are used to test the model. Other parameters: epochs $=200$, batch_size $=1$.

The error effect diagram of each variable using LSTM is shown in Figure 3. As can be seen from the Figure 3, except for the corn market price, the other three variables have a good fitting effect. After analyzing the corn market price sequence, it is found that the price fluctuation range of corn are between 1.2 yuan and 2.2 yuan. The price fluctuation range of the four variables is shown in Figure 3. Compared with the other three variables, the price fluctuation range of corn are the smallest and the degree of fluctuation are the most obvious, which is the main reason for the lowest prediction accuracy of corn market price.

This paper uses root mean square error (RMSE), mean absolute error (MAE), Mean absolute percentage error (MAPE) and R-squared (R2) as evaluation indexes to measure the performance of model prediction. Table 1 shows the evaluation results of LSTM, ANN, GRU and RNN in this experiment. The higher R2, the better the performance of the model. The smaller the value of the other three indicators, the better the performance of the model. It can be seen that LSTM has achieved best performance on four datasets. The R2 value of LSTM is the largest on all four data sets, reaching $0.88,0.67,0.99,0.99$ respectively. The RMSE value of the LSTM is the smallest on all four datasets, reaching $0.08,0.06,0.02,0.03$ respectively. MAE and MAPE are also the smallest on all four datasets. Therefore, LSTM has better advantages in predicting time series. This paper uses LSTM to predict four variables respectively.

\section{Experiments on Prediction of Soybean Price Trend}

The MLSTM-FCN and MALSTM-FCN have been tested on 35 datasets and performed well (Karim et al., 2019). Through a grid search, the authors found the optimal number of LSTM cells for each data set (ranging from 8 to 128). In this experiment, the initial batch size used is 128 , and the FCN block is composed of three 128-256-128 filter blocks. In the training phase, this paper sets the total number of epochs trained as 250 and the ratio of training set to test set as 8:2, in order to reduce overfitting. This paper uses Adam optimizer (Kingma \& Ba, 2014) to set the initial learning rate as $1 \mathrm{e}-3$ and the final learning rate as 1e-4. Before training, the data set was normalized and preprocessed so that it has zero mean value and unit variance. In addition, the learning rate decreases after every 100 times of learning. Note that this paper uses the Keras library (Chollet et al., 2015) with the TensorFlow backend to train the model. In order to highlight the performance of MALSTM-FCN, this paper compares MLSTM-FCN and MALSTM-FCN with LSTM-FCN and ALSTM-FCN, as shown in Figure 4.

Figure 4 shows the classification performance of the four models on soybean dataset. The accuracy of MALSTM-FCN is the highest among the four models, reaching 76.39\%. Then, MLSTMFCN has the second highest classification accuracy, reaching $73.61 \%$. The classification accuracy of LSTM-FCN is the lowest, reaching $72.22 \%$. It can be seen that the performance of MLSTM-FCN and MALSTM-FCN are better than that of LSTM-FCN and ALSTM-FCN. This is because the extruded layer and the exciter layer significantly improve the performance of the multivariate time series classifier by modeling the interdependence between channels. Furthermore, the accuracy of MALSTM-FCN is higher than the MLSTM-FCN model. At the same time, the accuracy of ALSTMFCN is higher than LSTM-FCN. This is because attention mechanism can improve the performance 
Figure 3. This is the error rendering of the four variables by LSTM model, where the blue line represents the original data set, the orange line represents the training value, and the green line represents the predicted value. Figure $3(\mathrm{a})$ is the error renderings of soybean purchase price, Figure 3(b) is the error renderings of corn market price, Figure 3(c) is the error renderings of soybean futures price, Figure $3(d)$ is the error renderings of soybean oil futures price.

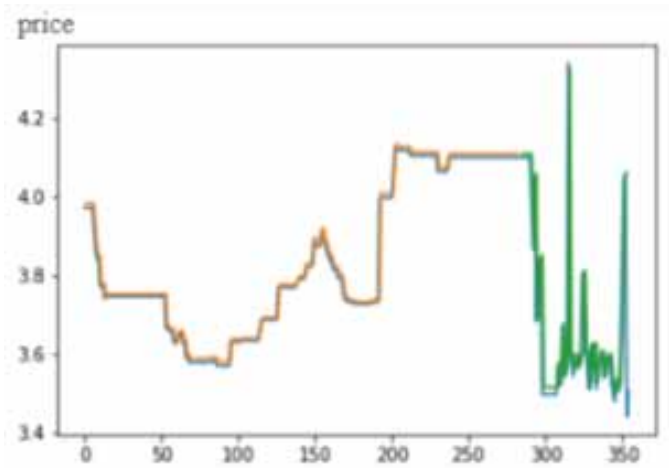

Figure 3(a)

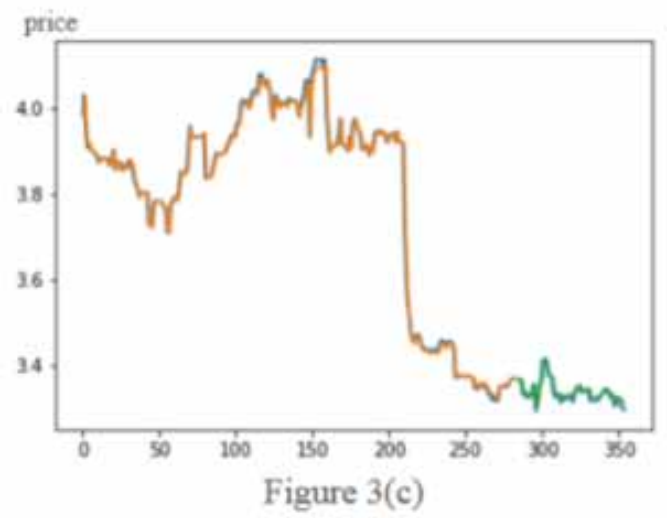

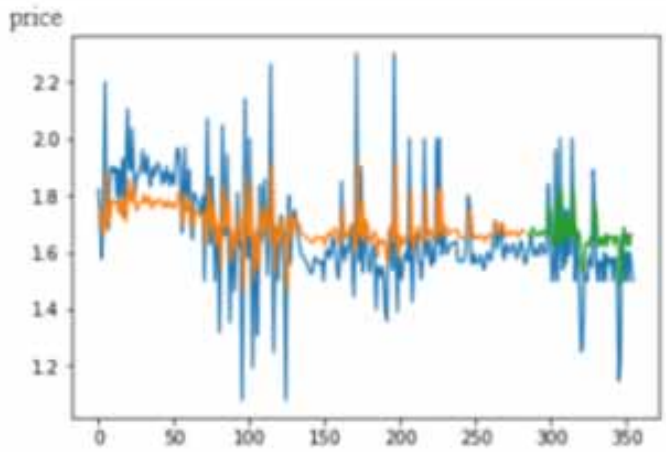

Figure 3(b)

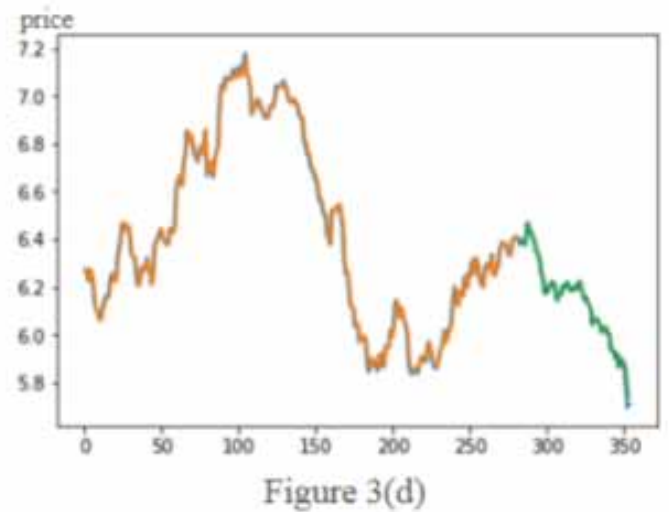

of the model. MALSTM-FCN is superior to the other three models, because it applies simultaneously squeeze-and-excite block and attention mechanism. The experiment results also prove this point.

Therefore, this paper verifies the feasibility of MALSTM-FCN model in classifying multivariate time series data. Using this classification algorithm could accurately determine which market level the soybean market price would be in and make corresponding measures in time.

When the soybean market is predicted to be at the risk level of recession, the government should take measure in time, such as making full use of financial support policy, increasing agricultural loans, and formulating credit policy designedly, meeting the capital demand of soybean production, soybean processing and soybean marketing. The government should strengthen macro policy guidance and create a good environment for industrial development.

When predicting that the soybean market is at risk level of prosperity, the government must strengthen the regulation of soybean production, take effective measures to reduce the production cost of soybean, and increase subsidies on soybean growers and enthusiasm for planting soybean, thereby guaranteeing the supply of soybean.

When the soybean market is predicted to be at risk level of recovery, the government should set up effective coordination mechanism and make the corresponding supporting policies, in order to improve the soybean market circulation environment and promote the development of soybean industry. Therefore, the government should pay attention to transfer of market information, establish 
Table 1. evaluation results of four variables in LSTM, ANN, GRU and RNN. The unit of measurement of prices in the table is yuan

\begin{tabular}{|l|l|l|l|l|l|}
\hline \multicolumn{1}{|c|}{ Data set } & \multicolumn{1}{|c|}{ Model } & \multicolumn{1}{c|}{ RMSE } & \multicolumn{1}{c|}{ MAE } & \multicolumn{1}{c|}{ MAPE } & \multicolumn{1}{c|}{ R2 } \\
\hline \multirow{5}{*}{ Soybean purchase price } & LSTM & 0.08 & 0.03 & 0.47 & 0.88 \\
\cline { 2 - 6 } & RNN & 0.1 & 0.04 & 1.11 & 0.82 \\
\cline { 2 - 6 } & GRU & 0.13 & 0.05 & 1.27 & 0.74 \\
\cline { 2 - 6 } & ANN & 0.34 & 0.17 & 32.61 & 0.85 \\
\hline \multirow{5}{*}{ Corn market price } & LSTM & 0.06 & 0.04 & 1.4 & 0.67 \\
\cline { 2 - 6 } & RNN & 0.07 & 0.05 & 3.06 & 0.62 \\
\cline { 2 - 6 } & GRU & 0.08 & 0.05 & 2.91 & 0.58 \\
\cline { 2 - 6 } & ANN & 0.06 & 0.04 & 24.53 & 0.64 \\
\hline \multirow{5}{*}{ Soybean futures price } & LSTM & 0.02 & 0.01 & 0.36 & 0.99 \\
\cline { 2 - 6 } & RNN & 0.03 & 0.02 & 0.36 & 0.95 \\
\cline { 2 - 6 } & GRU & 0.03 & 0.02 & 0.37 & 0.94 \\
\cline { 2 - 6 } & ANN & 0.05 & 0.04 & 3.8 & 0.95 \\
\hline \multirow{5}{*}{ Soybean oil futures price } & LSTM & 0.03 & 0.02 & 0.08 & 0.99 \\
\cline { 2 - 6 } & RNN & 0.04 & 0.03 & 0.35 & 0.97 \\
\hline & GRU & 0.03 & 0.12 & 0.04 & 0.96 \\
\cline { 2 - 6 } & ANN & & & 0.97 \\
\hline
\end{tabular}

professional market information service platform, providing farmers with soybean market present situation and foreign soybean market information and data.

Figure 4. Comparison of accuracy of four models on soybean dataset

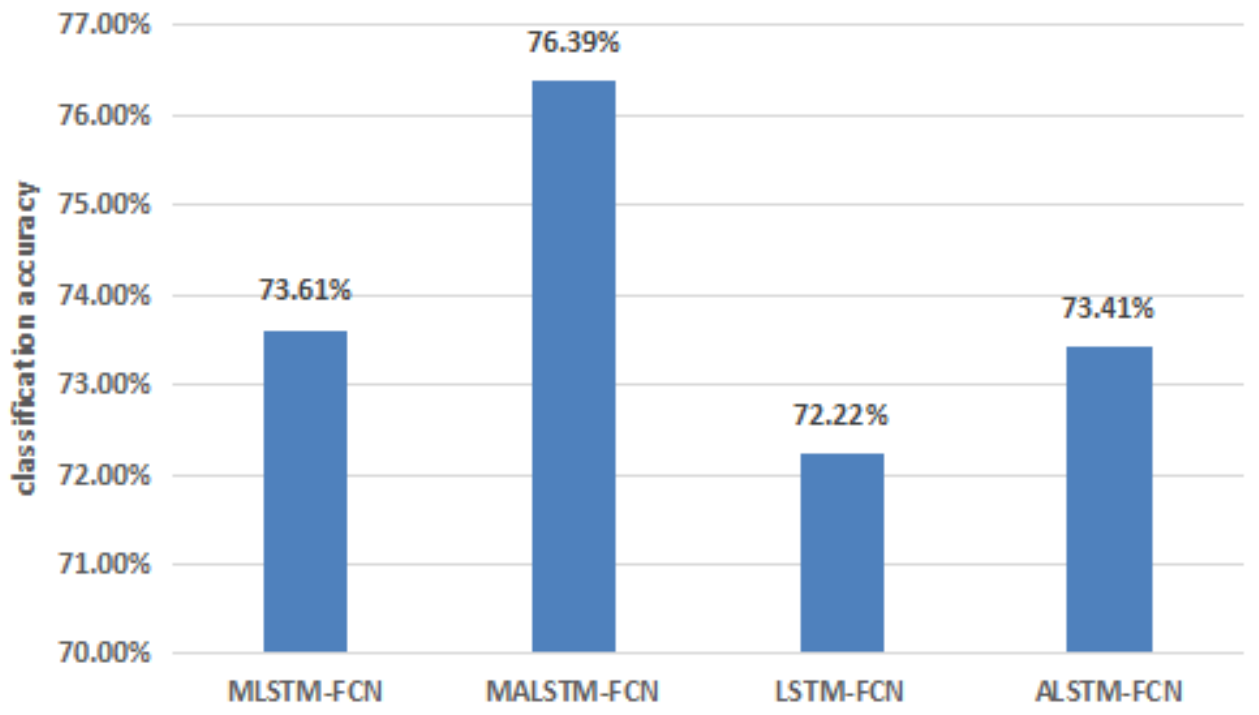


When predicting that the soybean market is at risk level of depression, the government should increase the special reserve of soybean, that is to purchase soybean at a reasonable price. The aim is to be able to adjust price if the soybean market price is too high.

Soybean is an important economic crop and occupies an important position in international trade. Soybean price is closely related to farmers living standard and agricultural market. Research on soybean price can enable related people to accurately grasp the market price trend, and then take beneficial measures. It is of great practical significance for stabilizing the market of agricultural products in China, improving the output value of animal husbandry, and promoting the development of agricultural economy. Therefore, it is very important to predict soybean market trend accurately.

\section{CONCLUSION AND FUTURE WORK}

This paper presents a soybean price trend prediction model. The model is a new combination with multistage model. First, this paper cites this conclusion from Deng and Sun (2019), that is, there are four patterns in soybean price. This paper takes these four patterns as the warning risk levels. Then, this paper uses LSTM to predict soybean purchase price, corn market price, soybean futures price and soybean-oil futures price, respectively. The experimental results show that LSTM can achieve better performance than ANN, RNN and GRU. This paper finally classifies the multivariate time series data consisting of four variables to find out which pattern the soybean market price is in. For classification experiments, MALSTM-FCN achieves great classification accuracy, compared with MLSTM-FCN, LSTM-FCN and ALSTM-FCN. In addition, we analyze the corresponding measures for each risk level. The study provides a new method for agricultural monitoring and early warning, which can provide certain reference for the price regulation of agricultural products market in China.

In the prediction experiment, the corn market price fitting is not good enough. The main reason is that the price fluctuation range of corn is between 1.2 yuan and 2.2 yuan. Compared with the other three variables, the price fluctuation range is the smallest and the degree of fluctuation is the most obvious. Therefore, the future work is to build better models for forecasting agricultural product price.

\section{ACKNOWLEDGMENT}

This work was supported by Harbin Applied Technology Research and Development Program under Grant 2017RAQXJ096. 


\section{REFERENCES}

Baek, Y., \& Kim, H. Y. (2018). ModAugNet: A new forecasting framework for stock market index value with an overfitting prevention LSTM module and a prediction LSTM module. Expert Systems with Applications, 113, 457-480. doi:10.1016/j.eswa.2018.07.019

Bahdanau, D., Cho, K., \& Bengio, Y. (2014). Neural machine translation by jointly learning to align and translate. Academic Press.

Baydogan, M. G., \& Runger, G. (2014). Learning a symbolic representation for multivariate time series classification. Data Mining and Knowledge Discovery, 29(2), 400-422. doi:10.1007/s10618-014-0349-y

Baydogan, M. G., \& Runger, G. (2015). Time series representation and similarity based on local autopatterns. Data Mining and Knowledge Discovery, 30(2), 476-509. doi:10.1007/s10618-015-0425-y

Cho, K., Merrienboer, B., Gulcehre, C., Bahdanau, D., Bougares, F., Schwenk, H., \& Bengio, Y. (2014). Learning phrase representations using RNN encoder-decoder for statistical machine translation. In Proceedings of the 2014 Conference on Empirical Methods in Natural Language Processing (pp.1724-1734). Association for Computational Linguistics. doi:10.3115/v1/D14-1179

CholletF. (2015). Keras. https://github.com/fchollet/keras

Cuturi, M., \& Doucet, A. (2011). Autoregressive kernels for time series. Academic Press.

David, S. A., Machado, J. A. T., Trevisan, L. R., Inácio, C. M. C., \& Lopes, A. M. (2017, March 11). Dynamics of commodities prices: Integer and fractional models. Fundamenta Informaticae, 151(1-4), 389-408. doi:10.3233/ FI-2017-1499

Deng, H., \& Sun, Y. (2019). Soybean price pattern Ddiscovery via toeplitz inverse covariance-based clustering. International Journal of Agricultural and Environmental Information Systems, 10(4), 1-17. doi:10.4018/ IJAEIS.2019100101

Drachal, K. (2019). Analysis of agricultural commodities prices with new Bayesian model combination schemes. Sustainability, 11(19), 11. doi:10.3390/su11195305

Graves, A. (2012). Supervised Sequence Labelling with Recurrent Neural Networks. Springer.

Hallac, D., Vare, S., Boyd, S., \& Leskovec, J. (2017). Toeplitz inverse covariance-based clustering of multivariate time series data. Proceedings of the 23rd ACM SIGKDD International Conference on Knowledge Discovery and Data Mining. doi:10.1145/3097983.3098060

He, P., Li, J., \& Zhang, D. (2017). Predicting Chinese soybean price based on APSO_SVR. Dadou Kexue, 36, 632-638.

Hu, J., Shen, L., \& Sun, G. (2017). Squeeze-and-excitation networks. Academic Press.

Ioffe, S., \& Szegedy, C. (2015). Batch normalization: Accelerating deep network training by reducing internal covariate shift. In Proceedings of the 32nd International Conference on International Conference on Machine Learning (vol. 37, pp.448-456). Academic Press.

Jaakkola, T., Diekhans, M., \& Haussler, D. (2000). A discriminative framework for detecting remote protein homologies. Journal of Computational Biology, 7(1-2), 95-114. doi:10.1089/10665270050081405 PMID: 10890390

Jain, A. K., Mao, J., \& Mohiuddin, K. M. (1996). Artificial neural networks: A tutorial. Computer, 29(3), 31-44. doi:10.1109/2.485891

Jiang, F. (2018). Applied research of non-linear method time series combination model in agricultural product price forecasting [Master dissertation, Lanzhou Jiaotong University]. China National Knowledge Infrastructure.

Karim, F., Majumdar, S., Darabi, H., \& Chen, S. (2018). LSTM fully convolutional networks for time series classification. IEEE Access: Practical Innovations, Open Solutions, 6, 1662-1669. doi:10.1109/ ACCESS.2017.2779939 
Karim, F., Majumdar, S., Darabi, H., \& Harford, S. (2019). Multivariate LSTM-FCNs for time series classification. Neural Networks, 116, 237-245. doi:10.1016/j.neunet.2019.04.014 PMID:31121421

Kingma, D., \& Ba, J. (2014). Adam: A method for stochastic optimization. Computer Science.

Li, C. (2014). Study on the price prediction of agro-products [Master dissertation, Hunan Agricultural University]. China National Knowledge Infrastructure.

Orsenigo, C., \& Vercellis, C. (2010). Combining discrete svm and fixed cardinality warping distances for multivariate time series classification. Pattern Recognition, 43(11), 3787-3794. doi:10.1016/j.patcog.2010.06.005

Patxi, O., Alberto, D.-O., Javier, D. S., Fernando, V., \& Mariluz, P., \& Basilio Sierra. (2020). Evolutionary LSTM-FCN networks for pattern classification in industrial processes. Swarm and Evolutionary Computation.

Pei, W., Dibeklioglu, H., Tax, D. M. J., \& van der Maaten, L. (2018). Multivariate time-series classification using the hidden-unit logistic model. IEEE Transactions on Neural Networks and Learning Systems, 29(4), 920-931. doi:10.1109/TNNLS.2017.2651018 PMID:28141534

Peng, Y., Liu, Y., \& Zhang, R. (2019). Modeling and analysis of stock price forecast based on LSTM. Computer Engineering and Application, 55, 209-212.

Qian, B. (2017). Research on agricultural product price forecasting model based on deep learning [Master dissertation, Anhui Agricultural University]. China National Knowledge Infrastructure.

Seto, S., Zhang, W., \& Zhou, Y. (2015). Multivariate time series classification using dynamic time warping template selection for human activity recognition. 2015 IEEE Symposium Series on Computational Intelligence, 1399-1406. doi:10.1109/SSCI.2015.199

Trottier, L., Giguère, P., \& Chaib-Draa B. (2016). Parametric exponential linear unit for deep convolutional neural networks. Academic Press.

Tsoi, A. C. (1998). Recurrent neural network architectures: An overview. Lecture Notes in Computer Science, 1387, 1-26. doi:10.1007/BFb0053993

Tuncel, K. S., \& Baydogan, M. G. (2018). Autoregressive forests for multivariate time series modeling. Pattern Recognition, 73, 202-215. doi:10.1016/j.patcog.2017.08.016

Wang, Y., Su, X., \& Guo, S. B. (2016). The optimal confidence intervals for agricultural products' price forecasts based on hierarchical historical errors. Entropy (Basel, Switzerland), 18(12), 439. doi:10.3390/e18120439

Wistuba, M., Grabocka, G., \& Schmidt-Thieme, L. (2015). Ultra-fast shapelets for time series classification. Academic Press.

Wu, G., Guo, Z., Li, L., \& Wang, C. (2020). Video abnormal detection combine FCN With LSTM. Journal of Shanghai Jiaotong University.

$\mathrm{Xu}, \mathrm{X} .$, \& Ma, K. (2018). Prediction and analysis of soybean price in China based on system dynamics. Dadou Kexue, 37, 787-793.

Zhang, D. Q., Zang, G. M., Li, J., Ma, K. P., \& Liu, H. (2018). Prediction of soybean price in China using QR-RBF neural network model. Computers and Electronics in Agriculture, 154, 10-17. doi:10.1016/j.compag.2018.08.016

Zheng, Y., Liu, Q., Chen, E., Ge, Y., \& Zhao, J. L. (2014). Time series classification using multi-channels deep convolutional neural networks. In International Conference on Web-Age Information Management (pp.298-310). doi:10.1007/978-3-319-08010-9_33 
Xu Zhiling was born in Henan Province, China, in 1995. She graduated from Zhengzhou University in 2017 with a bachelor's degree in management. During this period, she won the National Encouragement Scholarship. She began her master's degree in Northeast Agricultural University in 2018. Her research interest is in data analysis.

Hualing Deng is now a professor in College of Arts and Science in Northeast Agricultural University. She was born in 1965 in Heilongjiang Province, China. In 2003, she received his PhD in agricultural systems engineering from Northeast Agricultural University. Her current research interest is in applied mathematics.

Qiufeng Wu was born at Heilongjiang Province, China, in 1979. He received PhD in computer application technology from Harbin Institute of Technology, Harbin, China, in January 2014. He is working as associated professor in College of Arts and Sciences in Northeast Agricultural University. He is CCF member. His current research interests include machine learning, computer vision and smart agriculture. 